\title{
1 Rational design of proteins that exchange on functional timescales
}

2

3 James A. Davey, ${ }^{\dagger}$ Adam M. Damry, ${ }^{\dagger}$ Natalie K. Goto* \& Roberto A. Chica*

4

5 Affiliations:

6 Department of Chemistry and Biomolecular Sciences, University of Ottawa, Ottawa, Ontario,

$7 \quad \mathrm{~K} 1 \mathrm{~N} 6 \mathrm{~N} 5$, Canada

8

$9 \quad *$ Correspondence to: E-mail: rchica@uottawa.ca, ngoto@uottawa.ca

$10 \dagger$ These authors contributed equally to this work 


\section{Abstract}

13 Proteins are intrinsically dynamic molecules that can exchange between multiple

14 conformational states, enabling them to carry out complex molecular processes with extreme

15 precision and efficiency. Attempts to design novel proteins with tailored functions have mostly

16 failed to yield efficiencies matching those found in nature because standard methods do not allow

17 for the design of exchange between necessary conformational states on a functionally-relevant

18 timescale. Here, we develop a broadly-applicable computational method to engineer protein

19 dynamics that we term meta-multistate design. We used this methodology to design spontaneous

20 exchange between two novel conformations introduced into the global fold of Streptococcal protein

21 G domain $\beta 1$. The designed proteins, named DANCERs, for Dynamic And Native Conformational

22 ExchangeRs, are stably folded and exchange between predicted conformational states on the

23 millisecond timescale. The successful introduction of defined dynamics on functional timescales

24 opens the door to new applications requiring a protein to spontaneously access multiple

25 conformational states. 
Proteins have found widespread application in research, industry, and medicine because

they can mediate complex molecular processes with extreme precision and efficiency. Even so, continued engineering of proteins with tailored functions is essential to enable novel biotechnological applications. Computational protein design (CPD) has enjoyed considerable success in creating protein sequences that stably adopt a single targeted structure (1-5). However, attempts to use these methods to generate proteins that can carry out specific functions have mostly failed to match the efficiencies that are found in nature (6-9), suggesting that fundamental aspects of protein structure that are not currently considered in design strategies must be incorporated in order to create proteins that can approach the efficacy of naturally occurring systems. One such feature is dynamics, which have been shown to be essential for many complex protein functions

37 (10-13). The development of a general strategy for the rational design of protein sequences displaying predictable dynamic properties has great potential to expand the range and functionality

39 of designed proteins, paving the way to applications that are currently inaccessible using natural proteins.

The rational design of protein dynamics requires the prediction of sequences that can adopt

42 the necessary conformational states for exchange. The recent development of multistate design

43 (MSD) approaches applicable to large structural ensembles (14-16) has provided a method for the

44 evaluation of protein sequence energies in the context of a large number of possible conformational

45 states. Thus, MSD can in principle be used to assess the energy landscape of a target protein and

46 identify sequences that can exchange between distinct states. However, introduction of functionally

47 relevant conformational exchange into a stable protein fold is a difficult design problem as it

48 requires a priori knowledge of the structural features of the relevant conformational states for

49 dynamic exchange, including the endpoint structures and intermediate states that the protein must 
adopt as it undergoes this conformational transition, which are often unknown. In addition, the

51 multivariable optimization of sequences across many conformational states presents a significant

52 computational challenge, since sequences must be designed that not only satisfy stability

53 requirements for multiple target structures, but also yield an energy profile that would allow

54 exchange between structures to occur on a functionally relevant timescale.

Herein, we have developed a general procedure that addresses these challenges and enables

56 the rational design of protein dynamics, which we termed meta-MSD (Fig. 1). Meta-MSD enables

57 the evaluation of protein energy landscapes in order to predict sequences able to spontaneously

58 exchange between specific states. Unlike standard MSD methodologies where states are defined

59 by the user prior to calculation (e.g., target and off-target states), meta-MSD instead assigns the

60 identity of the states based on their structural characteristics after rotamer optimization, enabling

61 the unbiased prediction of the preferred state for each sequence, along with an evaluation of the

62 relative energies of every state that the sequence can stably adopt. We applied this methodology to

63 the design of sequences that adopt the global fold of Streptococcal protein G domain $\beta 1(\mathrm{G} \beta 1)$ and

64 spontaneously exchange between two conformations that have not been previously observed for

65 this fold. The designed dynamic G $\beta 1$ variants, termed DANCERs, for Dynamic And Native

66 Conformational ExchangeRs, were shown to be stably folded and to exchange between the

67 predicted conformational states on the millisecond timescale.

\section{Computational design of a protein energy landscape}

70 A dynamic protein that spontaneously interconverts between two distinct conformational

71 states adopts a continuum of unique configurations during exchange. However, the energy

72 landscape is complex and the range of configurations that are sampled over the course of exchange

73 cannot be completely defined. Nevertheless, it should be possible to engineer a user-defined 
74 exchange trajectory by identifying sequences that stabilize configurations having structural

75 characteristics postulated to facilitate this exchange. To simplify the exchange reaction coordinate,

76 the conformational landscape can be conceptually divided into three states: a major, a minor, and

77 a transition state (Fig. S1). In the context of this work, we treat each of these states as a collection

78 of unique configurations that we will refer to as microstates. Microstates are generated by

79 optimizing rotamers for predefined sequences on an ensemble of backbone templates using MSD,

80 which also returns an energy value for each microstate that reflects its predicted stability (Fig. 1,

81 panels I-III). Following MSD, microstates are partitioned into their corresponding states according

82 to their structural features (Fig. 1, panel IV), and the energy of each state is calculated from the

83 energy of its constituent microstates. Evaluation of relative energies between each state then allows

84 prediction of the exchange profile for each sequence, allowing identification of sequences that

85 would give rise to static or dynamic G $\beta 1$ folds (Fig. 1, panels V-VI). We call this framework meta-

86 MSD because both state and dynamic behavior are assigned after rotamer optimization by MSD.

87 Meta-MSD can be used to identify sequences that can stably populate the two target states, with a

transition state barrier that is small enough to allow interconversion between these two states, enabling the rational design of dynamics.

To validate our meta-MSD framework, we targeted the introduction of millisecond

91 timescale exchange into the G $\beta 1$ structure. Native $G \beta 1$ is rigid on this timescale (17), with a small

92 size (56 amino acids) that facilitates characterization of its dynamic properties at atomic resolution.

93 Additionally, G $\beta 1$ possesses a single tryptophan residue (Trp43) that in high-resolution structures

94 of G $\beta 1$ and its natively folded variants (18-28) exclusively occupies a single side-chain

95 conformation with $\chi_{1}$ and $\chi_{2}$ dihedrals of $-74 \pm 9^{\circ}$ and $+75 \pm 11^{\circ}$, respectively. We name this

96 conformation $+g(-)$ due to its positive $\chi_{2}$ dihedral angle and its gauche(-) $\chi_{1}$ dihedral (Fig. S2). In

$97 \mathrm{G} \beta 1$, the Trp43 side chain is mostly solvent inaccessible, making intimate contacts with several 
residues that comprise the hydrophobic core. This makes it an attractive target for the design of

99 conformational exchange, with one state being buried, and the other being excluded from the hydrophobic core in a solvent-exposed conformation that should be straightforward to distinguish spectroscopically. In addition, exchange between a core-buried and solvent-exposed state is expected to involve the disruption of side-chain interactions that should increase the kinetic barrier separating states, while not requiring large-scale changes in backbone structure that could prove kinetically inaccessible (29). Moreover, with exchange of the tryptophan side chain being set as our target for the design of dynamics, tryptophan side-chain dihedral angles provide a convenient metric for the assignment of microstates to one of the target states defined in our meta-MSD approach.

Using meta-MSD, we designed G $\beta 1$ sequences that could adopt the native fold and also undergo conformational exchange between a state where the Trp43 indole is solvent-exposed $[-g(+)]$ and a state where the indole is sequestered from the solvent in the hydrophobic core [$g(-)]$ (Fig. S3). Notably, we avoided selection of the native Trp43 conformation $[+g(-)]$ for the relative to non-native configurations (30). A final and particularly critical aspect of our conformational exchange design was the definition of an intermediate state with the Trp43 side chain in the $-t$ conformation, since this state is necessary to provide a model of transiently populated microstates that are sampled along the reaction coordinate. Use of the $-t$ conformation as a proxy of the transition state thus allowed estimation of kinetic barriers between states, enabling

118 the elimination of sequences predicted to stably adopt two end-states separated by large kinetic barriers that would not exchange on functionally relevant timescales. 
122

123

124

125

126

127

prepared using a combination of several template generation procedures (Fig. S4, Table S1, and SI Text). Using this ensemble, MSD was performed to optimize rotamers for a library of 1,296 G $\beta 1$ sequences comprising combinations of core-residue mutations (Fig. S5) that were previously reported to result in folded G $\beta 1$ variants (14). MSD thus yielded $>16$ million microstates and corresponding energies, allowing for approximation of the accessible conformational landscape of Trp43 in the native G $\beta 1$ fold.

Sequences having a Boltzmann-weighted average of MSD energies greater than that of the wild-type sequence are less likely to adopt a stable G $\beta 1$ fold (15) and were therefore eliminated from the meta-MSD analysis. For the remaining 195 sequences, each microstate was classified as being in a core-buried $[-g(-)]$, solvent-exposed $[-g(+)]$, or intermediate $[-t]$ state based on the $\chi_{1}$ and $\chi_{2}$ dihedrals of the Trp43 side chain. The energy of each of these states was determined for every sequence by taking the energy of the most stable microstate assigned to each state. State energies were used to construct an energy profile for each sequence (Fig. 1, panel V), enabling us to identify 35 sequences predicted to allow conformational exchange between the target coreburied and solvent-exposed conformations (SI Text), of which four were selected for experimental characterization (Table 1, DANCER proteins).

\section{Experimental characterization}

Although the four DANCER proteins each contained between five and six mutations, representing approximately $10 \%$ of the G $\beta 1$ total sequence length, they expressed as soluble monomers (Fig. S6), adopted the native G $\beta 1$ fold (Fig. S7), and were folded at room temperature (Fig. S8, Table S2). Chemical denaturation experiments (Fig. S9) could be fit to a two-state model with $m$-values similar to that of the wild type (Table S2), indicating a similar level of protein surface exposed to solvent upon unfolding (31). In addition, all DANCER variants have unfolding free 
energies that are $1.5 \mathrm{kcal} / \mathrm{mol}$ and higher (Table 1), confirming that they are stably folded at room

147 temperature. Solution NMR was used to assess the dynamic properties of DANCER proteins, with

$148{ }^{1} \mathrm{H}_{-}{ }^{15} \mathrm{~N}$ heteronuclear single quantum coherence (HSQC) spectra showing immediate evidence that

149 DANCER proteins exist in two distinct conformational states (Fig. S10). Specifically, spectra for DANCER-1, DANCER-2, and DANCER-3 all showed the presence of a minor species not seen in spectra of wild-type G $\beta 1$ (Fig. S11). The only exception was DANCER-0, which instead showed significant peak broadening, suggesting that it is dynamic on a faster timescale (32). acquired over a range of temperatures could be fit to kinetic and thermodynamic parameters of exchange for DANCER-1 and DANCER-3 (Table 1, Fig. S13), confirming that conformational exchange is occurring on the millisecond timescale. DANCER-1 exhibits approximately 10-fold faster exchange than DANCER-3, with an activation barrier that is $1.75 \mathrm{kcal} / \mathrm{mol}$ smaller in population of the minor state $(<10 \%)$ prevented quantitative measurement of kinetic parameters for this mutant.

To obtain structural evidence that the two conformations sampled by our dynamic G $\beta 1$ variants matched structural states predicted by meta-MSD, solution NMR was used to solve the structure of the major state of DANCER-2 (Fig. 3A, Table S3). As predicted, this structure shows 166 a native $\mathrm{G} \beta 1$ fold with $\chi_{1}$ and $\chi_{2}$ dihedrals for Trp43 that correspond to the solvent-exposed $-g(+)$ 167 conformation (Table 2). However, there was also a secondary network of low intensity NOEs 168 involving the Trp43 side chain that were not compatible with this structure, but could be used to 169 determine a structural model for the alternate, minor state (SI Text). According to this model (Fig. 
$3 \mathrm{~B}$, Table S3), the configuration of Trp43 in the minor state is in the core-buried $-g(-)$ state (Table

171 2), as predicted by meta-MSD. Taken together, these data demonstrate that we have successfully

172 designed a sequence that adopts the G $\beta 1$ fold while undergoing conformational exchange on a

173 millisecond timescale between two conformational states that have not previously been observed,

174 but were the targets of our design protocol.

To illustrate the reliability of our meta-MSD predictions, we also characterized the structure and dynamics of DANCER-1 and DANCER-3. While the exchange parameters for these mutants made it impractical to attempt structure determination, ${ }^{1} \mathrm{H}_{-}{ }^{15} \mathrm{~N}$ HSQC spectra of the major species

178 showed similarities with those of other structurally characterized variants, suggesting a high degree 179 of structural similarity with these states. Specifically, the DANCER-1 spectrum shows only small 180 chemical shift differences from that of DANCER-2 (Fig. 4A), suggesting that the major species of DANCER-1 also contains Trp43 in the solvent-exposed $-g(+)$ state. Likewise, the ${ }^{1} \mathrm{H}_{-}{ }^{15} \mathrm{~N}$ HSQC spectrum for DANCER-3 was highly similar to that of a variant that we determined to thermodynamically and kinetically favor the $-g(+)$ state as predicted by meta-MSD (Fig. 4B), G $\beta 1$ structure with Trp43 being in the solvent-exposed $-g(+)$ state. indole NH proton. DANCER-1 and DANCER-3 spectra show NOE correlations (Fig. S14) to similar regions of the protein as was observed in DANCER-2 (Fig. 4C), consistent with exchange 
core-buried state found in the wild-type structure $[+g(-)]$ (SI text, Fig. S15). Taken together, our

195 NMR results confirm that the Trp43 residues of DANCER-1 and DANCER-3 exchange between

the solvent-exposed $-g(+)$ and core-buried $-g(-)$ conformations that were the targets of our design,

197 and also suggest that exchange is achieved via a coordinated change in side-chain configurations

198 for a triad of aromatic residues (Phe34, Trp43, Phe45) in a process we have termed an aromatic

199 relay (SI text, Fig. S16).

200

201

\section{Discussion}

The meta-MSD framework described here enabled the rational design of G $\beta 1$ variants that spontaneously exchange between two predefined states on the millisecond timescale without the need for an external stimulus to induce exchange. To our knowledge, this work represents the first successful application of CPD to engineer a specific mode of conformational exchange into a stable protein fold. Although a previous CPD-based design generated a protein capable of reversible exchange between coiled-coil trimer and zinc-finger folds (8), this relied on the presence of a metal that was critical for the formation of the zinc finger structure. In that case, it was possible to design exchange by simultaneously minimizing the sum of the sequence energies across both folds. In contrast, to design conformational exchange between two states in the absence of a ligand or other external stimulus, we found that it was essential to explicitly consider both the relative energies

212 between the two target end-states $\left(\Delta \mathrm{E}_{\text {eq }}\right)$ and the barrier to conformational exchange $\left(\Delta \mathrm{E}^{\ddagger}\right)$.

213 Without estimation of both of these energy differences, it would not have been possible to 214 distinguish between dynamic (DANCER) and static (NERD) sequences (e.g., both $\Delta \mathrm{E}_{\text {eq }}$ and $\Delta \mathrm{E}^{\ddagger}$ values for DANCERs were lower than for NERDs and wild-type G $\beta 1$, Table 1). 
utilized in MSD approaches. This was of critical importance, since we found that the full

219 complement of seed structure and ensemble generation strategies used in our framework was

220 required to approximate the energy landscape of the designed exchange trajectory with enough

221 accuracy to predict DANCER variants (SI Text, Table S4). In addition, the large ensemble size

222 made it possible to design exchange in the absence of specific structures corresponding to each

223 end-state, in contrast with the metal-triggered conformational exchange that was previously

224 designed using available crystal structures as templates for the two end-states (8).

226 cannot be achieved via a single mutation and that instead dynamics is conferred through subtle

227 interactions across a network of residues. For example, the A34F mutation, which was previously

228 shown to induce dimerization of G $\beta 1$ without altering the Trp43 conformation $(27,33)$, is common

229 to all DANCER proteins and an integral component of the aromatic relay that underlies exchange

230 (Fig. S16). However, this mutation alone is not sufficient to introduce dynamics into the G $\beta 1$ fold,

231 since the variant NERD-S also possesses this mutation but does not undergo exchange on the

232 millisecond timescale (Table 1). Introduction of the conservative and isosteric I39L mutation into

233 the NERD-S sequence appears to be sufficient to introduce the targeted conformational exchange,

234 giving rise to the dynamic variant DANCER-3. These results highlight the challenges of attempting

235 to infer dynamics from simple sequence characteristics, and demonstrate the power of meta-MSD

236 to design conformational exchange into proteins even without prior knowledge of the mechanism

237 of exchange.

239 Conclusion

The meta-MSD framework presented here is in principle applicable to the design of specific 
design proteins with functions that rely on the ability to spontaneously access more than one

243 conformational state (e.g. open and closed states of an enzyme to facilitate substrate binding and

244 catalysis, respectively). Alternatively, meta-MSD could be used to enrich functionally relevant but

245 low occupancy states from an ensemble of dynamic configurations to improve function (34).

246 Moreover, while we have demonstrated the introduction of dynamics into a rigid protein,

247 dampening of dynamics should in principle also be possible, as demonstrated by our design of

248 NERD-C and NERD-S. This potential for meta-MSD to be used for the rigidification of highly

249 dynamic regions in proteins without adversely affecting the overall structure, in effect imitating

250 conformational selection in silico, opens the door to the design of proteins with a wider range of

251 functions than previously possible.

252

253 Data Availability. Structure coordinates have been deposited in the Protein Data Bank with 254 accession codes 5UB0 (NERD-C), 5UBS (NERD-S), 5UCE (major state of DANCER-2), and 255 5UCF (minor state of DANCER-2). NMR data has been deposited in the Biological Magnetic

256 Resonance Data Bank with accession codes 30220 (NERD-C), 30221 (NERD-S), 30222

257 (DANCER-2), 27030 (DANCER-0), 27031 (DANCER-1), and 27032 (DANCER-3).

\section{References}

260 1. B. I. Dahiyat, S. L. Mayo, De novo protein design: fully automated sequence selection.

$261 \quad$ Science 278, 82-87 (1997).

262 2. B. Kuhlman, G. Dantas, G. C. Ireton, G. Varani, B. L. Stoddard, D. Baker, Design of a 263 novel globular protein fold with atomic-level accuracy. Science 302, 1364-1368 (2003).

264 3. N. Koga, R. Tatsumi-Koga, G. Liu, R. Xiao, T. B. Acton, G. T. Montelione, D. Baker, 265 Principles for designing ideal protein structures. Nature 491, 222-227 (2012). 
4. E. Marcos, B. Basanta, T. M. Chidyausiku, Y. Tang, G. Oberdorfer, G. Liu, G. V. Swapna, R. Guan, D. A. Silva, J. Dou, J. H. Pereira, R. Xiao, B. Sankaran, P. H. Zwart, G. T. Montelione, D. Baker, Principles for designing proteins with cavities formed by curved beta sheets. Science 355, 201-206 (2017).

270 5. S. M. Malakauskas, S. L. Mayo, Design, structure and stability of a hyperthermophilic protein variant. Nat Struct Biol 5, 470-475 (1998).

6. L. Jiang, E. A. Althoff, F. R. Clemente, L. Doyle, D. Röthlisberger, A. Zanghellini, J. L. Gallaher, J. L. Betker, F. Tanaka, C. F. Barbas, 3rd, D. Hilvert, K. N. Houk, B. L. Stoddard, D. Baker, De novo computational design of retro-aldol enzymes. Science 319, 1387-1391 (2008).

276 7. S. Bjelic, L. G. Nivón, N. Çelebi-Ölçüm, G. Kiss, C. F. Rosewall, H. M. Lovick, E. L. Ingalls, J. L. Gallaher, J. Seetharaman, S. Lew, G. T. Montelione, J. F. Hunt, F. E. Michael, activity for the Morita-Baylis-Hillman reaction. ACS Chem Biol 8, 749-757 (2013). Sci U S A 109, 3790-3795 (2012).

10. G. Bhabha, J. Lee, D. C. Ekiert, J. Gam, I. A. Wilson, H. J. Dyson, S. J. Benkovic, P. E. Wright, A dynamic knockout reveals that conformational fluctuations influence the chemical step of enzyme catalysis. Science 332, 234-238 (2011). structures of proline isomerase essential for catalysis. Nature 462, 669-673 (2009). 
290

291

292

293

294

295

296

297

298

299

300

301

302

303

304

305

306

307

308

309

310

311

12. S. J. Kerns, R. V. Agafonov, Y. J. Cho, F. Pontiggia, R. Otten, D. V. Pachov, S. Kutter, L. A. Phung, P. N. Murphy, V. Thai, T. Alber, M. F. Hagan, D. Kern, The energy landscape of adenylate kinase during catalysis. Nat Struct Mol Biol 22, 124-131 (2015).

13. S. R. Tzeng, C. G. Kalodimos, Dynamic activation of an allosteric regulatory protein. Nature 462, 368-372 (2009).

14. B. D. Allen, A. Nisthal, S. L. Mayo, Experimental library screening demonstrates the successful application of computational protein design to large structural ensembles. Proc Natl Acad Sci U S A 107, 19838-19843 (2010).

15. J. A. Davey, R. A. Chica, Improving the accuracy of protein stability predictions with multistate design using a variety of backbone ensembles. Proteins 82, 771-784 (2014).

16. J. A. Davey, A. M. Damry, C. K. Euler, N. K. Goto, R. A. Chica, Prediction of Stable Globular Proteins Using Negative Design with Non-native Backbone Ensembles. Structure 23, 2011-2021 (2015).

17. K. A. Crowhurst, S. L. Mayo, NMR-detected conformational exchange observed in a computationally designed variant of protein Gbeta1. Protein Eng Des Sel 21, 577-587 (2008).

18. S. Butterworth, V. Lamzin, D. Wigley, J. Derrick, K. Wilson, Anisotropic refinement of a protein G domain at 1.1 ångstrom resolution. The Protein Databank in Europe (accessed 13.11. 2012). Available online at: http://www. ebi. ac. uk/pdbe, (1998).

19. J. P. Derrick, D. B. Wigley, The third IgG-binding domain from streptococcal protein G. An analysis by X-ray crystallography of the structure alone and in a complex with Fab. $J$ Mol Biol 243, 906-918 (1994). 
20. T. Gallagher, P. Alexander, P. Bryan, G. L. Gilliland, Two crystal structures of the B1 immunoglobulin-binding domain of streptococcal protein $\mathrm{G}$ and comparison with NMR. Biochemistry 33, 4721-4729 (1994).

21. A. M. Gronenborn, D. R. Filpula, N. Z. Essig, A. Achari, M. Whitlow, P. T. Wingfield, G. M. Clore, A novel, highly stable fold of the immunoglobulin binding domain of streptococcal protein G. Science 253, 657-661 (1991).

22. B. J. Wylie, L. J. Sperling, A. J. Nieuwkoop, W. T. Franks, E. Oldfield, C. M. Rienstra, Ultrahigh resolution protein structures using NMR chemical shift tensors. Proc Natl Acad Sci U S A 108, 16974-16979 (2011).

23. J. H. Tomlinson, V. L. Green, P. J. Baker, M. P. Williamson, Structural origins of pHdependent chemical shifts in the B1 domain of protein G. Proteins 78, 3000-3016 (2010).

24. D. J. Wilton, R. B. Tunnicliffe, Y. O. Kamatari, K. Akasaka, M. P. Williamson, Pressureinduced changes in the solution structure of the GB1 domain of protein G. Proteins 71, 1432-1440 (2008).

25. P. Strop, A. M. Marinescu, S. L. Mayo, Structure of a protein G helix variant suggests the importance of helix propensity and helix dipole interactions in protein design. Protein Sci 9, 1391-1394 (2000).

26. T. Saio, K. Ogura, M. Yokochi, Y. Kobashigawa, F. Inagaki, Two-point anchoring of a lanthanide-binding peptide to a target protein enhances the paramagnetic anisotropic effect. Journal of biomolecular NMR 44, 157-166 (2009).

27. J. Jee, R. Ishima, A. M. Gronenborn, Characterization of specific protein association by 15N CPMG relaxation dispersion NMR: the GB1(A34F) monomer-dimer equilibrium. $J$ Phys Chem B 112, 6008-6012 (2008). 
28. J. Kuszewski, A. M. Gronenborn, G. M. Clore, Improving the packing and accuracy of NMR structures with a pseudopotential for the radius of gyration. Journal of the American Chemical Society 121, 2337-2338 (1999).

29. J. R. Lewandowski, M. E. Halse, M. Blackledge, L. Emsley, Protein dynamics. Direct observation of hierarchical protein dynamics. Science 348, 578-581 (2015).

30. J. A. Davey, R. A. Chica, Optimization of rotamers prior to template minimization improves stability predictions made by computational protein design. Protein Sci $\mathbf{2 4}, 545-$ $560(2015)$.

31. J. K. Myers, C. N. Pace, J. M. Scholtz, Denaturant $m$ values and heat capacity changes: relation to changes in accessible surface areas of protein unfolding. Protein Sci 4, 2138 2148 (1995).

I. R. Kleckner, M. P. Foster, An introduction to NMR-based approaches for measuring protein dynamics. Biochim Biophys Acta 1814, 942-968 (2011).

348 33. J. Jee, I. J. Byeon, J. M. Louis, A. M. Gronenborn, The point mutation A34F causes dimerization of GB1. Proteins 71, 1420-1431 (2008).

34. E. Campbell, M. Kaltenbach, G. J. Correy, P. D. Carr, B. T. Porebski, E. K. Livingstone, role of protein dynamics in the evolution of new enzyme function. Nature chemical biology

35. Y. Zhang, J. Skolnick, Scoring function for automated assessment of protein structure template quality. Proteins 57, 702-710 (2004). PROSPECTOR_3 threading algorithm. Proteins 56, 502-518 (2004). 
37. T. Gallagher, P. Alexander, P. Bryan, G. L. Gilliland, 2 Crystal-Structures of the B1 Immunoglobulin-Binding Domain of Streptococcal Protein-G and Comparison with Nmr. Biochemistry. 33, 4721-4729 (1994).

38. Chemical Computing Group Inc. (Chemical Computing Group Inc., 1010 Sherbooke St. West, Suite \#910, Montreal, QC, Canada, H3A 2R7, 2012).

39. P. Labute, Protonate3D: Assignment of ionization states and hydrogen coordinates to macromolecular structures. Proteins-Structure Function and Bioinformatics 75, 187-205 (2009).

40. I. W. Davis, W. B. Arendall, 3rd, D. C. Richardson, J. S. Richardson, The backrub motion: how protein backbone shrugs when a sidechain dances. Structure 14, 265-274 (2006).

41. F. Lauck, C. A. Smith, G. F. Friedland, E. L. Humphris, T. Kortemme, RosettaBackrub-a web server for flexible backbone protein structure modeling and design. Nucleic Acids Research 38, W569-W575 (2010).

42. S. G. Nash, A survey of truncated-Newton methods. J. Comput. Appl. Math. 124, 45-59 (2000).

43. J. Wang, P. Cieplak, P. A. Kollman, How well does a restrained electrostatic potential (RESP) model perform in calculating conformational energies of organic and biological molecules? Journal of Computational Chemistry 21, 1049-1074 (2000).

44. R. A. Chica, M. M. Moore, B. D. Allen, S. L. Mayo, Generation of longer emission wavelength red fluorescent proteins using computationally designed libraries. Proc Natl Acad Sci U S A 107, 20257-20262 (2010).

45. B. D. Allen, S. L. Mayo, Dramatic performance enhancements for the FASTER optimization algorithm. J Comput Chem 27, 1071-1075 (2006). 
46. B. D. Allen, S. L. Mayo, An efficient algorithm for multistate protein design based on FASTER. J Comput Chem 31, 904-916 (2010).

47. R. L. Dunbrack, F. E. Cohen, Bayesian statistical analysis of protein side-chain rotamer preferences. Protein Science 6, 1661-1681 (1997).

48. S. L. Mayo, B. D. Olafson, W. A. Goddard, Dreiding - a Generic Force-Field for Molecular Simulations. J. Phys. Chem. 94, 8897-8909 (1990).

49. B. I. Dahiyat, S. L. Mayo, Probing the role of packing specificity in protein design. Proc Natl Acad Sci U S A 94, 10172-10177 (1997).

50. T. Lazaridis, M. Karplus, Discrimination of the native from misfolded protein models with an energy function including implicit solvation. Journal of Molecular Biology 288, 477 487 (1999).

51. E. K. Koepf, H. M. Petrassi, M. Sudol, J. W. Kelly, WW: An isolated three-stranded antiparallel beta-sheet domain that unfolds and refolds reversibly; evidence for a structured hydrophobic cluster in urea and $\mathrm{GdnHCl}$ and a disordered thermal unfolded state. Protein Science : A Publication of the Protein Society 8, 841-853 (1999).

52. F. Delaglio, S. Grzesiek, G. W. Vuister, G. Zhu, J. Pfeifer, A. Bax, NMRPipe: A multidimensional spectral processing system based on UNIX pipes. Journal of biomolecular NMR 6, 277-293 (1995).

53. B. A. Johnson, R. A. Blevins, NMR View: A computer program for the visualization and analysis of NMR data. Journal of biomolecular NMR 4, 603-614 (1994).

54. D. Wishart, B. Sykes, F. Richards, The chemical shift index: a fast and simple method for the assignment of protein secondary structure through NMR spectroscopy. Biochemistry 31, 1647-1651 (1992). 
404 55. N. A. Farrow, O. Zhang, J. D. Forman-Kay, L. E. Kay, A heteronuclear correlation experiment for simultaneous determination of $15 \mathrm{~N}$ longitudinal decay and chemical exchange rates of systems in slow equilibrium. Journal of biomolecular NMR 4, 727-734 (1994).

56. Y. Shen, F. Delaglio, G. Cornilescu, A. Bax, TALOS+: a hybrid method for predicting protein backbone torsion angles from NMR chemical shifts. Journal of biomolecular NMR 44, 213-223 (2009).

57. P. Güntert, in Protein NMR Techniques, A. K. Downing, Ed. (Humana Press, Totowa, NJ, 2004), pp. 353-378.

58. C. A. Smith, T. Kortemme, Predicting the tolerated sequences for proteins and protein interfaces using RosettaBackrub flexible backbone design. PloS one 6, e20451 (2011).

59. J. A. Davey, R. A. Chica, Multistate Computational Protein Design with Backbone Ensembles. Methods Mol Biol 1529, 161-179 (2017).

60. I. W. Davis, A. Leaver-Fay, V. B. Chen, J. N. Block, G. J. Kapral, X. Wang, L. W. Murray, contacts and structure validation for proteins and nucleic acids. Nucleic Acids Res 35, W375-383 (2007).

424 62. Y. J. Huang, R. Powers, G. T. Montelione, Protein NMR Recall, Precision, and F-measure Retrieval Statistics. Journal of the American Chemical Society 127, 1665-1674 (2005). 


\section{Supplementary Materials:}

429 Supplementary Text

430 Figs. S1 to S16

431 Tables S1 to S4

432

\section{Acknowledgments}

434 R.A.C. acknowledges grants from the Natural Sciences and Engineering Research Council of 435 Canada (NSERC), the Ontario Research Fund, and the Canada Foundation for Innovation. N.K.G. 436 acknowledges a grant from NSERC. J.A.D. is the recipient of an Ontario Graduate Scholarship and 437 A.M.D. is the recipient of a NSERC postgraduate scholarship. We acknowledge Dr. Glenn Facey, 438 Dr. Yves Aubin, and Simon Sauvé for assistance with NMR experiments, as well as Dr. Yun Mou 439 for helpful discussions.

\section{Author Contributions}

442 J.A.D. and A.M.D. performed the experiments and analyzed data. N.K.G. and A.M.D. designed

443 NMR experiments and analyzed data. J.A.D. and R.A.C. designed computational experiments. All 444 authors wrote the manuscript. 
Table 1. Predicted and Experimental Properties of G $\beta 1$ variants

\begin{tabular}{|c|c|c|c|c|c|c|c|c|c|}
\hline \multirow[b]{2}{*}{ Protein } & \multirow[b]{2}{*}{ Mutations } & \multicolumn{3}{|c|}{ Meta-MSD Predictions } & \multirow{2}{*}{$\begin{array}{c}\text { Stability } \\
\Delta \mathrm{GU}^{\mathrm{d}} \\
\text { (kcal/mol) }\end{array}$} & \multicolumn{4}{|c|}{ Exchange $^{e}$} \\
\hline & & Behavior $^{\text {a }}$ & $\begin{array}{c}\Delta \mathrm{E}_{\mathrm{eq}}^{\mathrm{b}} \\
(\mathrm{kcal} / \mathrm{mol})\end{array}$ & $\begin{array}{c}\Delta \mathrm{E}^{\ddagger \mathrm{c}} \\
(\mathrm{kcal} / \mathrm{mol})\end{array}$ & & $\begin{array}{l}k_{-1}{ }^{f} \\
\left(s^{-1}\right)\end{array}$ & $\begin{array}{l}k_{1}{ }^{g} \\
\left(s^{-1}\right)\end{array}$ & $\begin{array}{c}\Delta \mathrm{G}^{\ddagger \mathrm{h}} \\
(\mathrm{kcal} / \mathrm{mol})\end{array}$ & $\begin{array}{c}\Delta \mathrm{G}_{\mathrm{eq}}^{\mathrm{i}} \\
(\mathrm{kcal} / \mathrm{mol})\end{array}$ \\
\hline Wild type & & $+g(-)$ & 8.6 & 15.2 & $4.1 \pm 0.2$ & & & & \\
\hline DANCER-0 & $\mathrm{Y} 3 \mathrm{~F} / \mathrm{L} 5 \mathrm{~A} / \mathrm{L} 7 \mathrm{I} / \mathrm{A} 34 \mathrm{~F} / \mathrm{V} 39 \mathrm{I}$ & $-g(+) \leftrightarrow-g(-)$ & 0.9 & 7.8 & $1.5 \pm 0.2$ & & & & \\
\hline DANCER-1 & Y3F/L5A/L7I/A34F/V39L/V54I & $-g(-) \leftrightarrow-g(+)$ & 3.7 & 8.4 & $2.2 \pm 0.1$ & $30 \pm 10$ & $110 \pm 50$ & $18.9 \pm 0.3$ & $0.3 \pm 0.1$ \\
\hline DANCER-2 & Y3F/L5A/L7I/A34F/V39L & $-g(+) \leftrightarrow-g(-)$ & 1.3 & 9.4 & $1.7 \pm 0.1$ & $\mathrm{j}$ & $\mathrm{j}$ & $\mathrm{j}$ & $1.4 \pm 0.7$ \\
\hline DANCER-3 & Y3F/L7I/A34F/V39L/V54I & $-g(-) \leftrightarrow-g(+)$ & 2.9 & 13.7 & $2.0 \pm 0.3$ & $3.9 \pm 0.2$ & $23 \pm 5$ & $20.65 \pm 0.08$ & $1.3 \pm 0.3$ \\
\hline NERD-S & Y3F/L7I/A34F/V39I/V54I & $-g(+)$ & 4.3 & 14.7 & $2.7 \pm 0.1$ & & & & \\
\hline NERD-C & Y3F/L7I/F30L/V39I & $+g(-)$ & 12.2 & 15.3 & $4.0 \pm 0.3$ & & & & \\
\hline
\end{tabular}

$450 \quad{ }^{b}$ Energy difference between the two lowest energy states

$451 \quad{ }^{\mathrm{c}}$ Energy barrier to conformational exchange (see SI text for more detail)

$452{ }^{\mathrm{d}}$ Free energy of unfolding determined by chemical denaturation with guanidium chloride at $25^{\circ} \mathrm{C}$

453 e Kinetic parameters $\left(k_{1}, k_{-1}, \Delta \mathrm{G}^{\ddagger}\right)$ reported at $15^{\circ} \mathrm{C}, \Delta \mathrm{G}_{\text {eq }}$ at $25^{\circ} \mathrm{C}$.

$454 \quad$ f Rate constant for exchange from major to minor state

$455 \mathrm{~g}$ Rate constant for exchange from minor to major state

$456{ }^{\mathrm{h}}$ Energy barrier for exchange from major to minor state

457 i Free energy difference between major and minor states

$458 \quad{ }^{j}$ Exchange peaks were observed but could not be quantified 
Table 2. Comparison of predicted and experimental structures

\begin{tabular}{|c|c|c|c|c|}
\hline Protein & TM-score to 1 PGA ${ }^{a}$ & $\begin{array}{c}\text { Predicted } \\
\text { Trp43 Conformation }\end{array}$ & $\begin{array}{c}\text { Experimental } \chi_{1} \\
\left({ }^{\circ}\right)\end{array}$ & $\begin{array}{c}\text { Experimental } \chi_{2} \\
\left({ }^{\circ}\right)\end{array}$ \\
\hline \multicolumn{5}{|l|}{ DANCER-2 } \\
\hline Major species & 0.67 & $-g(+)$ & $+75 \pm 2$ & $-74 \pm 1$ \\
\hline Minor Species & 0.66 & $-g(-)$ & $-95 \pm 1$ & $-110 \pm 2$ \\
\hline \multicolumn{5}{|l|}{ Static G及1 variants } \\
\hline NERD-S & 0.66 & $-g(+)$ & $+54 \pm 4$ & $-89 \pm 2$ \\
\hline NERD-C & 0.85 & $+g(-)$ & $-84 \pm 4$ & $+80 \pm 4$ \\
\hline
\end{tabular}

${ }^{a} \mathrm{TM}$-score has a value between 0 and 1 , where 1 indicates a perfect match between two structures. Two proteins with a TM-score greater than 0.5 are considered to adopt the same fold $(35,36)$. 


\section{- Multistate Design}

I. Ensemble of Backbone Templates

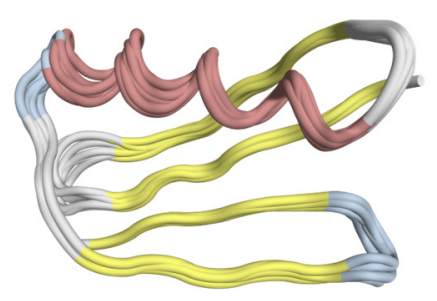

II. Rotamer Configurations

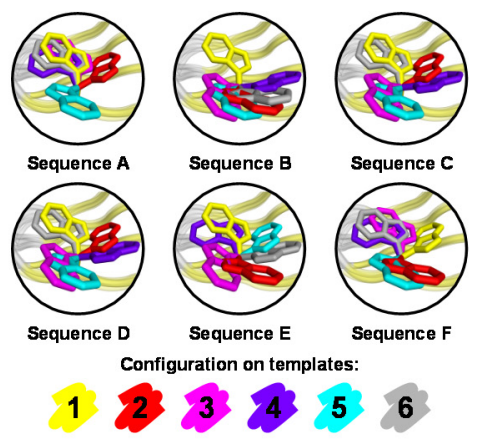

III. Sequence Energy

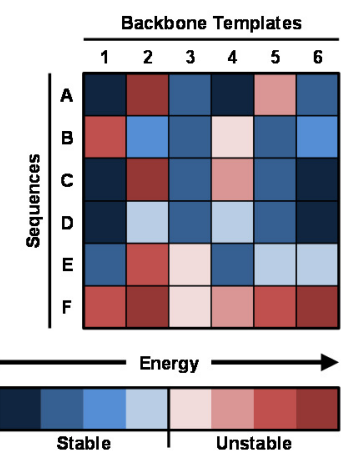

- Analysis

IV. Geometry Evaluation
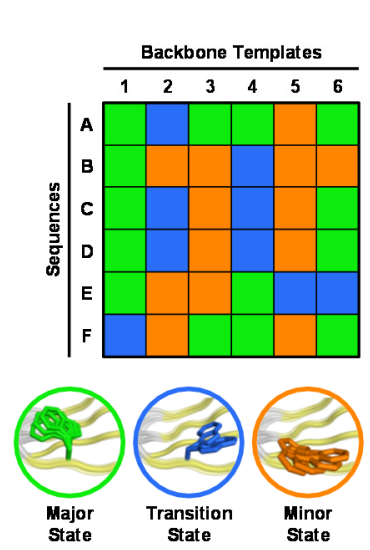

465

466

467

468

469

470

471

472

473

474

Figure 1. Meta-MSD. Multistate design (MSD) with an ensemble of backbone templates approximating the conformational landscape for dynamic exchange between targeted states (I) is used to generate microstates by solving the lowest energy rotamer configuration for each sequence on each backbone template (II). MSD also returns an energy value for each microstate that reflects its predicted stability (III). A geometry-based analysis of the rotamer-optimized microstates is performed (IV), allowing assignment of each microstate to major, minor or transition state in the energy landscape (V). Prediction of conformational dynamics is then done based on an evaluation of the relative energies of these states (VI). For a sequence to be predicted as dynamic, all three 
475 states must be stable, with an energy profile that is compatible with exchange (e.g., sequence D).

476 Sequences A, B, and C are predicted to be static because they either stabilize a single state or cannot

477 stabilize the transition state required for exchange. Sequence E is also predicted to be static because

478 it stabilizes only one endpoint state. Sequence $\mathrm{F}$ is predicted to be unfolded because it is unstable

479 on all states.

480 


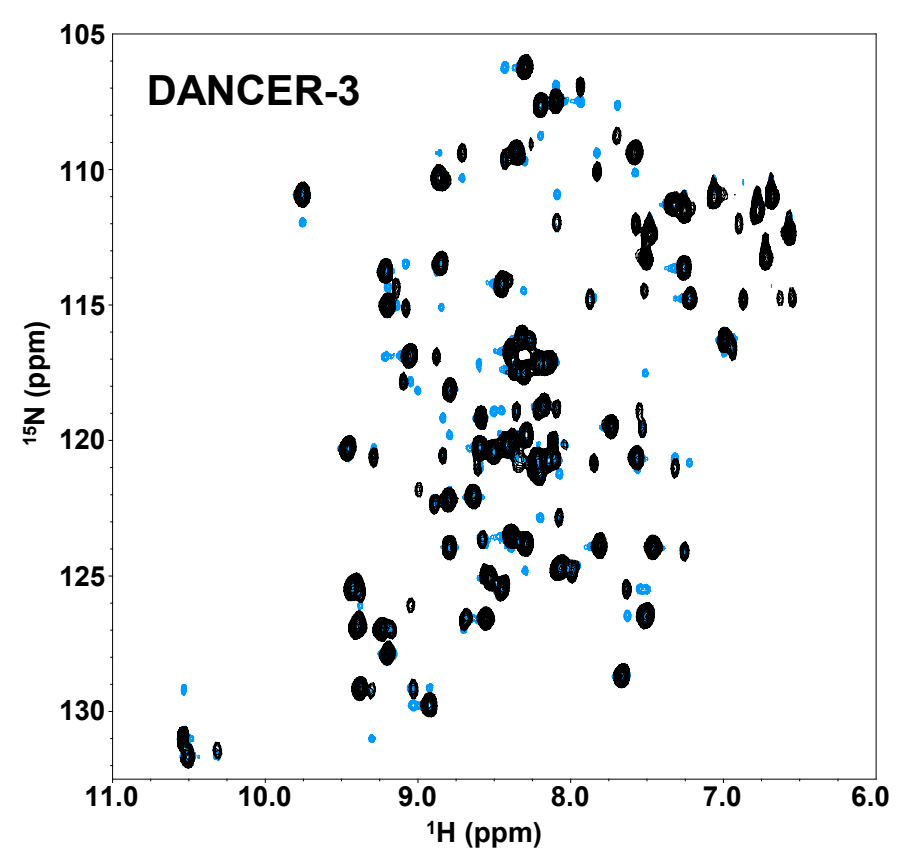

483

484 Figure 2. ${ }^{1} \mathbf{H}-{ }^{15} \mathbf{N}$ ZZ-Exchange spectrum for DANCER-3. ZZ-Exchange spectrum (blue) is 485 shown overlaid with ${ }^{1} \mathrm{H}^{-15} \mathrm{~N}$ HSQC spectrum (black) to highlight the presence of exchange peaks. 
A

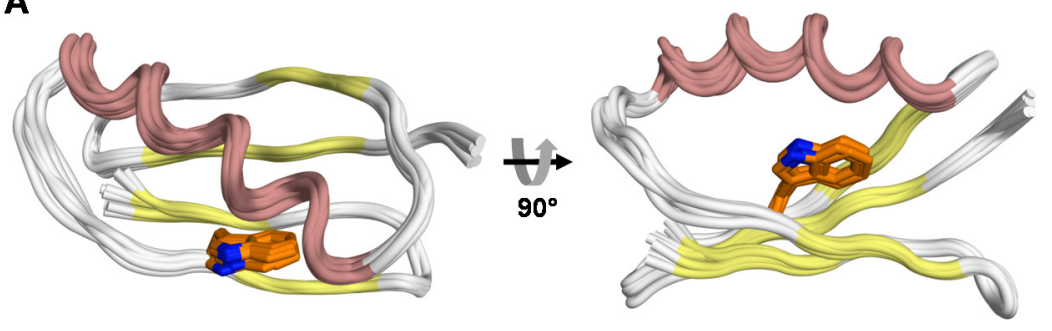

B
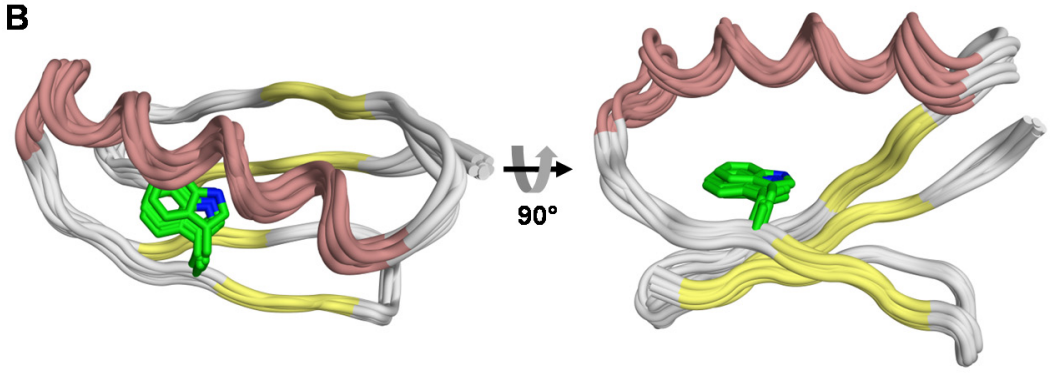

C
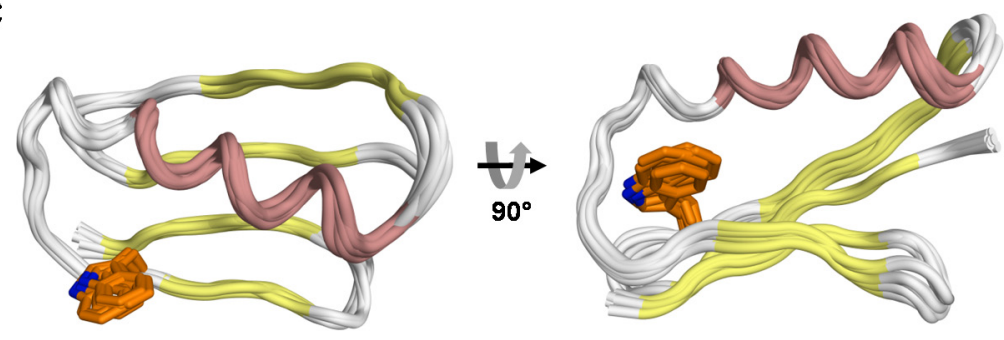

489 Figure 3. Solution structures of Gß1 variants. NMR ensembles for (A) DANCER-2 major

490 species, (B) DANCER-2 minor species, and (C) NERD-S. The minor species of DANCER-2 is a

491 model generated using NOESY data that excluded a small subset of peaks from the automatic NOE

492 assignment process that could be unambiguously assigned to the major species (SI Text). The

493 Trp43 side chain is shown as sticks.

494

495 

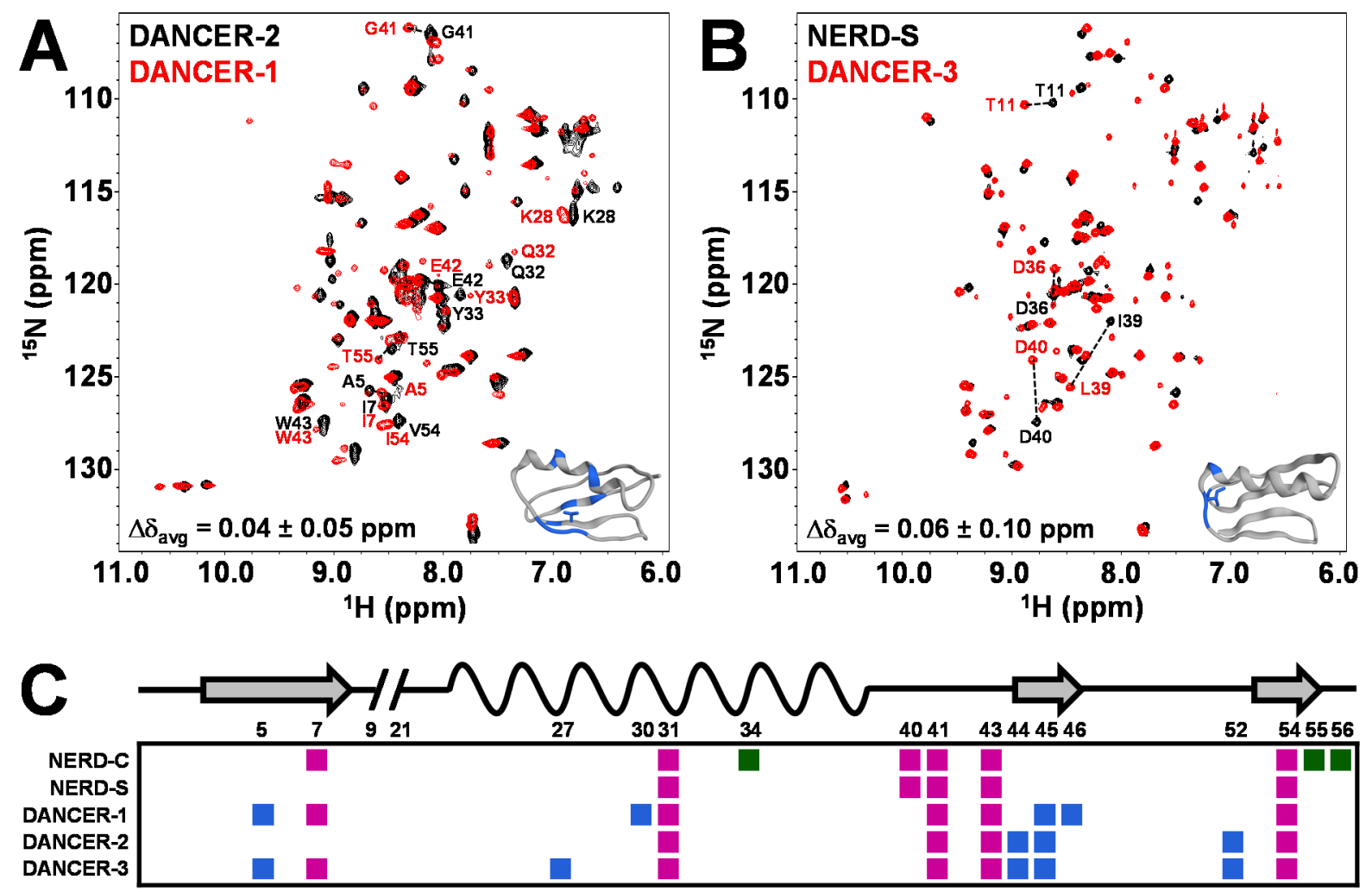

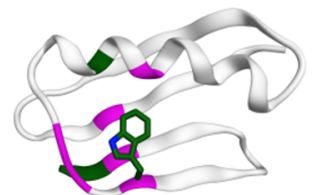

NERD-C

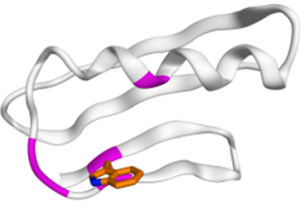

NERD-S

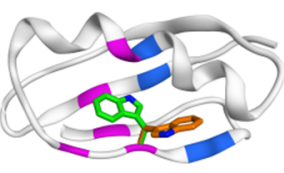

DANCER-1

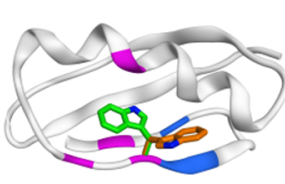

DANCER-2

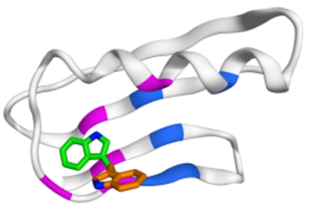

DANCER-3

Figure 4. Structural analysis of DANCER-1 and DANCER-3. (A) Superimposed ${ }^{1} \mathrm{H}-{ }^{15} \mathrm{~N}-\mathrm{HSQC}$

spectra of DANCER-2 and DANCER-1 reveal high structural similarity between major states.

Residues showing significant average amide shift differences $\left(\Delta \delta>\Delta \delta_{\text {avg }}+1 \sigma\right)$ are labeled and

highlighted in blue on the inset DANCER-2 structure. These residues are all proximal to the single

503 Summary of NOE correlations involving the Trp43 indole N-H shown on a position map

504 (secondary structure elements on top) and on each structure. Correlations are colored green, blue, 
506 conformation(s) consistent with observed NOEs are shown for each structure. Included in this 507 analysis is the solution NMR structure of NERD-C (Non-Exchanging Rigid Design with a Core508 buried Trp43 conformation, SI Text), which adopts the native $+g(-)$ configuration (Fig. S15, Table 509 2). NERD-C shows several unique indole N-H NOE correlations that are not observed in any of 510 the DANCER variants, confirming that this state is not sampled by the DANCER proteins. 